Journal of

Applied

Crystallography

ISSN 0021-8898

Editor: Gernot Kostorz

\title{
A simple model for dynamic small-angle $X$-ray diffraction in colloidal crystals
}

Alex G. F. de Beer and Andrei V. Petukhov

Copyright $\odot$ International Union of Crystallography

Author(s) of this paper may load this reprint on their own web site provided that this cover page is retained. Republication of this article or its storage in electronic databases or the like is not permitted without prior permission in writing from the IUCr. 
Journal of

\section{Applied \\ Crystallography}

ISSN 0021-8898

Received 2 June 2006

Accepted 6 November 2006

(C) 2007 International Union of Crystallography Printed in Singapore - all rights reserved

\section{A simple model for dynamic small-angle X-ray diffraction in colloidal crystals}

\author{
Alex G. F. de Beer* $¥$ and Andrei V. Petukhov
}

van 't Hoff Laboratory for Physical and Colloid Chemistry, Debye Institute, University of Utrecht, Padualaan 83508 TB Utrecht, The Netherlands. Correspondence e-mail: debeer@mf.mpg.de

A simple model is presented that allows calculation of the small-angle X-ray diffraction patterns of perfect colloidal crystals. The model is based on the Wentzel-Kramers-Brillouin approximation and permits a straightforward evaluation of multibeam interactions. Results are illustrated by several examples.

\section{Introduction}

Colloidal suspensions display spontaneous formation of crystals driven by the excluded-volume entropy (Hoover \& Ree, 1968; Pusey \& van Megen, 1986). They provide simple model systems for atomic and molecular phase transitions (Hoover \& Ree, 1968; Pusey \& van Megen, 1986; Pusey et al., 1989; Schaetzel \& Ackerson, 1993; Harland \& van Megen, 1997; Zhu et al., 1997; Davidchack \& Laird, 2000; Polson et al., 2000; Cheng et al., 2002; Auer \& Frenkel, 2003; Pronk \& Frenkel, 2003) and have recently re-entered the spotlight because of their possible photonic applications (Imhof \& Pine, 1997; Asher et al., 1998; Wijnhoven \& Vos, 1998; Blanco et al., 2000; Rengarajan et al., 2001; Vlasov et al., 2001; Velikov, 2002; Hoogenboom et al., 2003; Yethiraj \& van Blaaderen, 2003).

In the case of hard spheres, the formed crystals commonly show a random hexagonal close-packed (r.h.c.p.) structure (Sanders, 1968; Liu et al., 1993; Verhaegh et al., 1995; Zhu et al., 1997; Dux \& Versmold, 1997; Pronk \& Frenkel, 1999; Kegel \& Dhont, 2000; Martelozzo et al., 2002; Petukhov et al., 2002, 2003; Versmold et al., 2002), even though calculations indicate that colloidal hard spheres should prefer a face-centered cubic (f.c.c.) structure (Bolhuis et al., 1997).

The local structure and ordering of these crystals can be investigated using confocal microscopy (van Blaaderen et al., 1997; Verhaegh et al., 1995; Gasser et al., 2001; Elliot et al., 2001), while the long-range order can be conveniently investigated using scattering techniques. In particular, small-angle X-ray diffraction (SAXD) can be used to characterize the internal three-dimensional structure of colloidal crystals (Vos et al., 1997; Wijnhoven et al., 2001; Petukhov et al., 2002, 2003; Versmold et al., 2002; Megens \& Vos, 2001), as well as to probe their corresponding long-range order parameters (Petukhov et al., 2002). Even though the diffraction angles are extremely small, SAXD patterns can be clearly resolved using synchrotron sources that allow for sufficient angular resolution, of the order of a microradian (Drakopoullos et al., 2005; Petukhov et al., 2006). In comparison with light scattering (Cheng et al.,

$\$$ Present address: Max Planck Insitute for Metal Research, Heisenbergstrasse 3, 70569 Stuttgart, Germany.
2002; Henderson \& van Megen, 1998; Zhu et al., 1997; Dux \& Versmold, 1997; Kegel \& Dhont, 2000), the most important advantage of X-rays is their intrinsically low refractive index contrast (the difference in index of refraction is of the order of $\left.10^{-6}\right)$. As a result, the occurrence of multiple scattering is reduced significantly and a much wider range of scattering vectors is available with $\mathrm{X}$-rays. This significantly broadens the range of crystals that can be studied with SAXD including photonic band-gap materials which strongly interact with visible light.

As a direct result of the low contrast, scattering is weak and the Rayleigh-Gans-Debye (RGD) theory often suffices to describe the SAXD intensities. However, in single colloidal crystals possessing perfect periodic order over large distances, strong diffracted waves can be built so that the RGD can no longer be applied and the more complicated dynamic theory (James, 1965; Zachariasen, 1946), which accounts for the interactions between incident and diffracted waves, has to be used. In particular, two clear dynamic effects have been observed: diffraction spots of magnitude comparable with the zeroth-order beam (Petukhov et al., 2004) along with the appearance of so-called secondary Bragg rods at Bragg-rodforbidden diffraction orders (Petukhov et al., 2003). Here we present a simple model that allows the straightforward evaluation of the SAXD patterns even in the strongly dynamic diffraction limit. To illustrate the results, SAXD patterns were simulated for a number of examples.

\section{Model}

In the hard X-ray $(>10 \mathrm{keV})$ range, the index of refraction of matter is determined mainly by electron density (Feigin \& Svergun, 1987). For a typical colloidal system [for instance silica spheres immersed in a hydrocarbon solvent, as described by Petukhov et al. (2004)], the phase contrast (the real part of the difference in indices of refraction) is of the order of $10^{-6}$, while the absorption contrast (the imaginary part) is two orders of magnitude smaller (see http://www-cxro.lbl.gov/ optical_constants/). As a result, phase contrast dominates over absorption contrast in this energy range. 
Moreover, with the small phase contrast, all refraction can be neglected. Under these circumstances, the diffraction of a plane electromagnetic wave on a sample can be modeled by the Wentzel-Kramers-Brillouin (WKB) approximation. This model, which found prior application in quantum mechanics (Sakurai, 1994), visible optics (Deirmendjian, 1957) and nonlinear optics (Roke et al., 2004), treats the experimental sample as a pure phase object. Thus, when irradiated with a plane wave, the wave emerging from the sample is assumed to still be completely parallel, but modulated by a phase factor proportional to the pass lengths of the wave through different compounds (with different, non-complex, indices of refraction). In practice, the WKB approximation is especially suitable to SAXD with its low contrast and very small diffraction angles (typically of the order of $10^{-3}-10^{-4} \mathrm{rad}$ ).

The emerging phase-modulated wavefront (see also Fig. 1) is then allowed to propagate over a large distance. When analyzed in the Fraunhofer (i.e. far-field) approximation, a diffraction image is obtained that is proportional to the (squared modulus of a) two-dimensional Fourier transform of the field immediately after the sample. Since both phase modulation and the Fourier transform are energy conserving, the total power of the direct beam together with all the diffracted peaks is equal to the energy of the incoming beam.

In this work, we will use the WKB approximation to form a diffraction model that describes diffraction from a (perfect) r.h.c.p. lattice, consisting of perfectly round identical spheres. We will look at the diffraction pattern for a situation where the incident beam is perpendicular to the close-packed layers, and a situation where the incident beam is parallel to the closepacked layers. We are able to derive the diffraction pattern analytically for one orientation (the perpendicular orientation), though we are still able to draw important conclusions

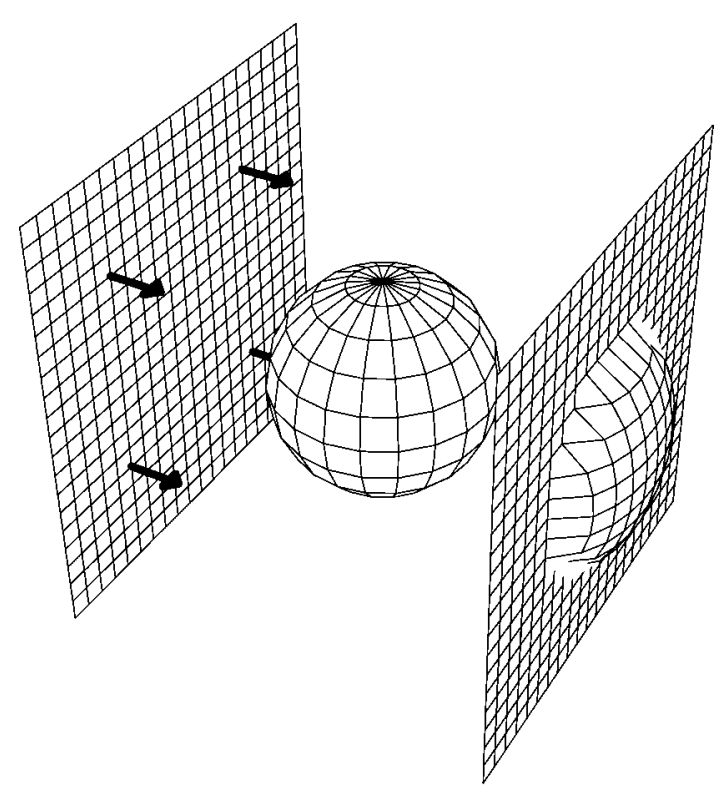

Figure 1

Schematic view of a wavefront hitting a sphere. As the wavefront travels through the sphere, it develops an advanced phase compared with the rest of the wave, which manifests as a wavefront distortion. from the results for the other orientation. Of course, for any orientation other than the two mentioned, the diffraction patterns can still be simulated numerically, though that is beyond the scope of this paper.

\section{Perpendicular orientation}

We first consider an X-ray wave perpendicular to the closepacked layers of a r.h.c.p. crystal. When the incident plane wave travels through a single layer, every point in the plane wave becomes multiplied by phase factors that are proportional to the amount of matter encountered in this layer. These phase factors are typical for the type of layer $(A, B$ or $C)$. Should the (now modified) plane wave encounter another layer of the same type, every point is multiplied by the same phase factors as before. Therefore, in the perpendicular orientation, the exact sequence of layer types is of no importance; only the number of occurrences of each layer type is. The phase modulation can be described as

$$
\tau(x, y)=\varphi_{A}^{N_{A}}(x, y) \varphi_{B}^{N_{B}}(x, y) \varphi_{C}^{N_{C}}(x, y),
$$

where $\varphi_{A, B, C}$ is the phase modulation for a given point $(x, y)$ in the close-packed layer $A, B$ or $C$, and $N_{A, B, C}$ is the number of layers of the respective type.

If we now calculate the diffraction patterns for each of the single layer types, we can calculate the full diffraction pattern by convoluting these results with each other. Furthermore, because each layer type is shifted over a well defined distance and direction with respect to the other layer types, it will suffice to calculate the Fourier transform for one type and add the proper shift factors for the other types. Similar to classical diffraction theory, the structure of a single plane can be described as a convolution of a unit cell and a (two-dimensional) lattice. The Fourier transform of the lattice will yield the reciprocal-lattice points, while the Fourier transform of the unit cell will yield the form factor, which holds the information we are interested in.

\subsection{Scattering from a single sphere}

As a simple example, we first calculate the scattering pattern of a single sphere. To maintain a clear connection with the following examples, the pattern is calculated for a stack of hexagonal layers in the same lateral position $A$ and containing $N_{A}$ layers. The only difference is that the convolution with the lattice is not performed and the Fourier components are calculated for continuously varying values $q$ of the scattering wavevector $\mathbf{q}$. The result thus represents the Fourier pattern of a two-dimensional unit, which is simply a line of $N_{A}$ spheres obscuring one another. This, in turn, is equivalent to the scattering field of a single sphere with an $N_{A}$-fold contrast of the refraction index. We should also note that in most of the simulation results in this section, the contrast is somewhat exaggerated in comparison with the conditions encountered in an X-ray scattering experiment. 
For such a sphere, the maximum phase difference an incoming plane wave incurs is [following the approach taken by Roke et al. (2004)]

$$
\psi=2 K_{0} N_{A} R_{\mathrm{s}} \Delta n,
$$

where $K_{0}$ is the value of the wavevector of the incident wave, $R_{\mathrm{S}}$ is the radius of a sphere and $\Delta n$ is the difference in index of refraction between sphere and solvent.

For a $10 \mathrm{keV}$ beam, $K_{0}$ is $5 \times 10^{9} \mathrm{~m}^{-1}$, so for spheres with radii of the order of $100 \mathrm{~nm}$ and an index of refraction contrast of $\Delta n \simeq 1 \times 10^{-6}$, a number of layers $N_{A}$ of the order of $10^{2}-$ $10^{3}$ are needed for diffraction to leave the RGD regime.

When we express the phase modulation field for a single particle in a transformed coordinate system by mapping the top hemisphere of the particle to the projection plane by the coordinates $\theta$ (for azimuth) and $\varphi$ (for altitude), the scattered electric field strength becomes

$$
\begin{aligned}
E_{A}\left(q_{R}\right)= & R_{\mathrm{s}}^{2} \int_{0}^{2 \pi} \int_{0}^{\pi / 2}[\exp (i \psi \sin \varphi)-1] \exp \left[i q_{R} R_{\mathrm{s}} \cos \theta \cos \varphi\right] \\
& \times \sin \varphi \cos \varphi \mathrm{d} \varphi \mathrm{d} \theta,
\end{aligned}
$$
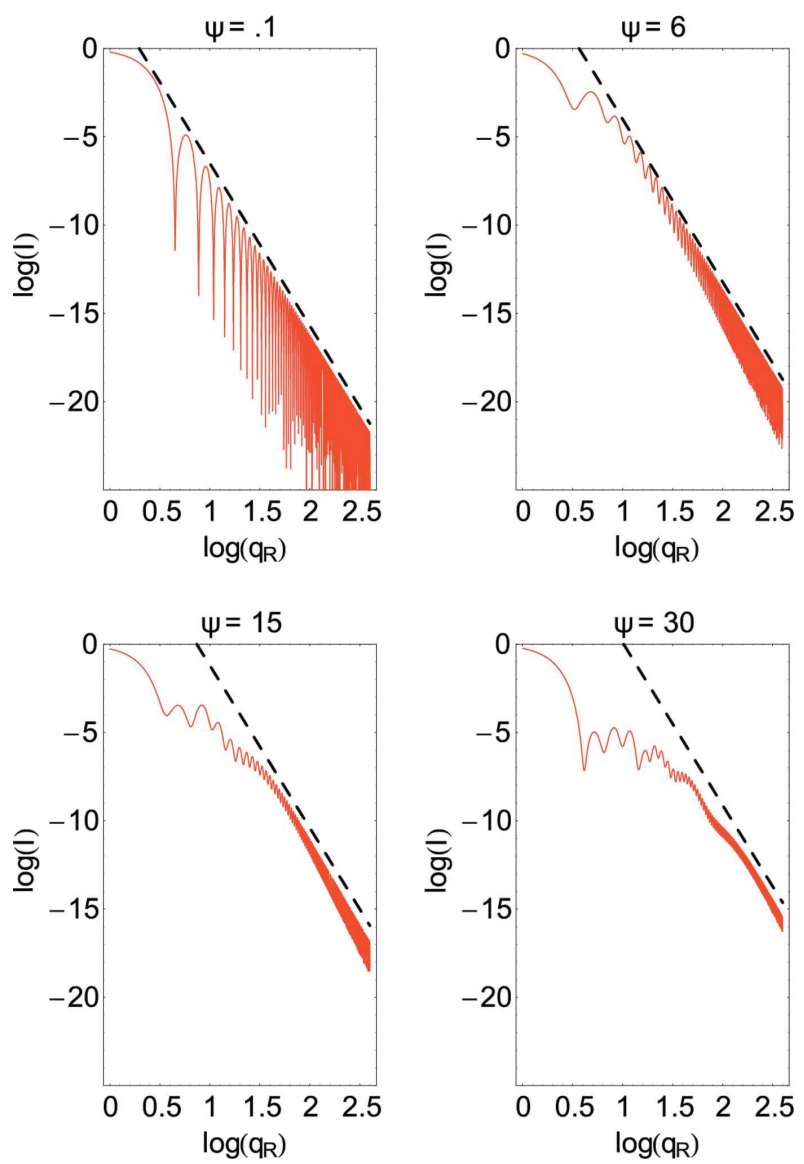

Figure 2

Log-log plots of scattered intensity as a function of scattering angle for different values of $\psi$. It can clearly be seen that, although a higher $\psi$ causes a later onset of the $q_{R}^{-4}$ dependence, it is still visible for values that are at the limit of the validity of our current model. with $q_{R}$ the radially symmetric diffraction vector. By expanding both exponentials, integrating and re-summing the results, this equation can be solved and written as (for a similar treatment, see van de Hulst, 1981)

$$
\begin{aligned}
E_{A}\left(q_{R}\right)= & 2 \pi \frac{R_{\mathrm{s}}^{2}}{q_{R} R_{\mathrm{s}}} \sum_{n=1}^{+\infty} \frac{(i \psi)^{n}}{n !} \Gamma\left(\frac{n}{2}+1\right)\left[\left(\frac{2}{q_{R} R_{\mathrm{s}}}\right)^{1 / 2}\right]^{n} \\
& \times J_{n / 2+1}\left(q_{R} R_{\mathrm{s}}\right),
\end{aligned}
$$

with $\Gamma$ being Euler's gamma function and $J_{n}$ the Bessel function of the first kind. This expression represents the scattering from a single sphere in a solvent, so the model should, in the low-contrast limit, correspond to classical scattering models. For small values of $\psi$, all terms beyond the first of this equation can be neglected. Furthermore, the first term can be rewritten as

$$
E_{A}\left(q_{R}\right)=2 \pi i \psi R_{\mathrm{s}}^{2}\left[\frac{\sin q_{R} R_{\mathrm{s}}-q_{R} R_{\mathrm{s}} \cos q_{R} R_{\mathrm{s}}}{\left(q_{R} R_{\mathrm{s}}\right)^{3}}\right],
$$

which is the RGD expression for scattering from a single spherical particle (see also van de Hulst, 1981).

In the RGD regime, at large $q_{R}$ the mean scattered intensity diminishes $\left(q_{R}^{-4}\right)$ according to Porod's law. Equation (4) seems to follow this behavior even at very high contrast. This is clearly visible in a log-log plot presented in Fig. 2. Despite the significant modifications of the dependence at smaller angles, it switches to a straight-line decay with a slope of -4 . Furthermore, when equation (4) is tested against Mie theory calculations (van de Hulst, 1981), good correspondence is observed for values of $\psi$ up to 20 (see Fig. 3).
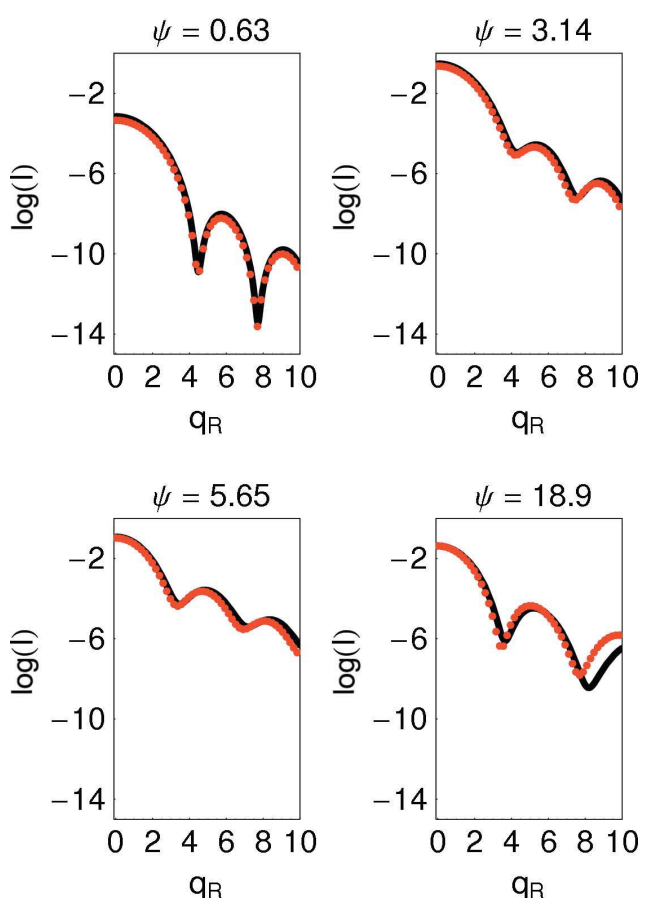

Figure 3

Comparison of Mie scattering (red dots) and the current model (black line) for different values of $\psi$. Only at higher values of $\psi$ can a significant deviation be observed. 


\section{research papers}

\subsection{An f.c.c. crystal}

From the expression of a single sphere, it is now possible to derive the expressions for single close-packed layers of the three types $(A, B, C)$ present in r.h.c.p. stackings, by multiplying equation (4) with the reciprocal lattice for a closepacked layer. For the $B$ and $C$ type layers, an additional phase factor is added because of the real-space translation of these layers compared with the $A$ type.

As noted before, the exact sequence of layers is irrelevant in the WKB approximation and only the amount of layers of each type is of importance. As a result, the layers in the r.h.c.p. structure (e.g. $A B A C B C B$ ) can be rearranged in such a way that a single f.c.c. crystal is formed, with any surplus layers added separately at the end (for the previous example: $A B C A B C B)$. Finally, the f.c.c. part can then be restacked into three zones with one layer type each $(A A B B C C B)$. We can now calculate the diffraction pattern for an f.c.c. crystal and convolute the result with the expressions of the surplus layers to obtain the r.h.c.p. result. The f.c.c. diffraction pattern, in turn, can be calculated by convoluting the expressions for the three layers types. The numbers of layers of each layer type are equal, and the translational shift of the $B$ or $C$ layer with respect to the $A$ layer is $\pm 2 R_{\mathrm{s}} /\left(3^{1 / 2}\right)$, which in the Fourier domain gives rise to a phase factor $\exp ( \pm i 2 \pi / 3)$ so that the expression for the convolution [adopting a two-dimensional $(h k)$ Miller index notation] becomes

$$
\begin{aligned}
E_{\mathrm{FCC} h k}= & \sum_{h^{\prime \prime}=-\infty}^{\infty} \sum_{k^{\prime \prime}=-\infty}^{\infty} E_{A\left(h^{\prime \prime} k^{\prime \prime}\right)} \exp \left[i(2 \pi / 3)\left(h^{\prime \prime}-k^{\prime \prime}\right)\right] \\
& \times \sum_{h^{\prime}=-\infty}^{\infty} \sum_{k^{\prime}=-\infty}^{\infty} E_{A\left(\left[h^{\prime}-h^{\prime \prime}\right]\left[k^{\prime}-k^{\prime \prime}\right]\right)} \\
& \times \exp \left\{-i(2 \pi / 3)\left[\left(h^{\prime}-h^{\prime \prime}\right)-\left(k^{\prime}-k^{\prime \prime}\right)\right]\right\} E_{A\left(\left[h-h^{\prime}\right]\left[k-k^{\prime}\right]\right)}
\end{aligned}
$$

where $E_{A h k}$ is the value of equation (4) for the diffraction vector corresponding to index $(h k)$. The occurrence of the factor $\exp (i 2 \pi / 3)$ is such that for any $h-k$ not divisible by three, the value of $E_{\mathrm{FCC} h k}$ is zero, which is a well known property of the f.c.c. diffraction.

From the series expansion of equation (6), a diffraction spectrum of the f.c.c. crystal can be simulated. Simulation of the relative intensity of the 11 peak with respect to the primary, undiffracted beam at increasing values of $\psi$ shows that there is a point where the primary beam is depleted up to a point where the intensity of the 11 diffraction is of comparable magnitude (see Fig. 4). As mentioned before, the WKB approximation conserves energy, which in this case means that a significant drain on the primary beam occurs, because all six first-order diffraction spots have the same intensity.

Furthermore, as the primary beam is depleted with increasing $\psi$, the first-order diffraction spots also shows a frustrated growth, due to both multiple scattering to higher orders and the lack of 'supply' from the lowered intensity of the primary beam. Finally, if we look at the evolution of the diffraction pattern with increasing $\psi$, we note that before the
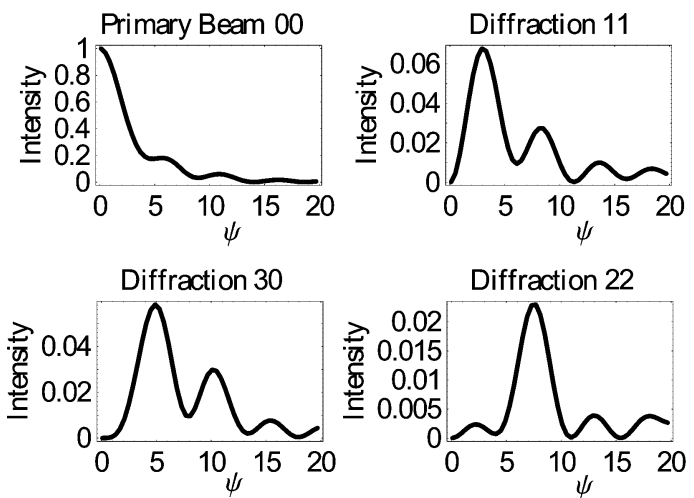

Figure 4

The intensities of the four lowest order non-zero f.c.c. diffraction peaks with increasing $\psi$. As $\psi$ increases, relatively more energy is diffracted to higher orders

11 diffraction reaches an intensity comparable with the primary beam, the 30 reaches an intensity comparable with the 11 diffraction. In the overall picture we notice, as expected, an increasing amount of energy scattered to higher orders.

\subsection{An r.h.c.p. crystal}

The final step in describing diffraction from an r.h.c.p. stacking is to convolute the result for f.c.c. stacking with the expressions for the surplus layers mentioned above. Typically, the number of surplus layers will be very small, which means that for these layers the condition $\psi<1$ is observed. As a result, the RGD equation (5) can be used as the expression for the scattering. One can see that the intensities of the peaks existing in an f.c.c. diffraction pattern are hardly altered due to the dominant contribution of the 00 value of the surplus layers. The intensities of the peaks that are zero in the f.c.c. expression, on the other hand, increase quadratically with the number of surplus layers, as can be seen in Fig. 5.

\section{Parallel orientation}

If we now switch the setup in order for the beam to be incident parallel to the close-packed layers, another picture is obtained. The structure of the crystal is not as straightforward to describe as in the previous orientation. It is possible, however,
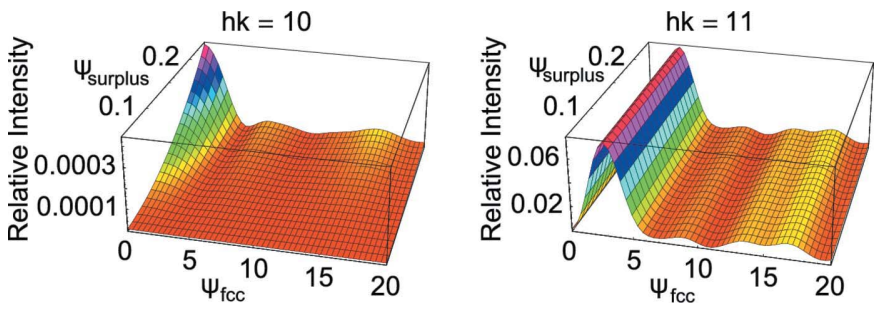

Figure 5

The influence of a number of surplus layers on the diffraction intensity of the 10 and 11 diffraction peaks. The intensity of the 10 peak increases with quadratic dependence on the phase difference caused by the surplus layers, while the influence of the surplus layers on the 11 peak is negligible. 


\section{research papers}

to calculate an average diffraction pattern based on the chances of a certain layer stacking occurring. For this, we create a variation on the method described by Wilson (1949).

The unit element can be defined as the upper half of one close-packed layer combined with the lower half of the neighboring layer. Because these elements are fully periodical in one direction, we expect well defined diffraction orders in this direction (to which we will refer as the intralayer direction). In the other direction (to which we will refer as interlayer direction), however, the lattice is only semi-periodical, and we expect Bragg rods to occur.

In our crystal, six combinations of half-layer pairs are possible ( $A B, B C, C A, B A, C B$ and $A C$, where the first letter denotes the lower half of a half-layer pair). Of these six combinations, two $(B C$ and $C A)$ are translations of the pair $A B$, while the remaining three $(B A, C B$ and $A C)$ are mirror images of the previous three.

In Fourier space, the translation leads to a phase factor $\exp ( \pm i n 2 \pi / 3)$ (where $n$ is the diffraction order in the intralayer direction). Mirroring leads to the inversion of one of the two coordinates, i.e. $F_{A B}\left(q_{x}, q_{y}\right)=F_{A C}\left(-q_{x}, q_{y}\right)$, where $F_{A B}$ is the diffraction pattern for unit element $A B$, and $x$ and $y$ are the intralayer and interlayer directions, respectively.

Because the crystal is periodical in the intralayer direction, we can suffice with picking only those values of $q_{x}$ that correspond with a diffraction spot. The diffraction intensity for the entire crystal then becomes

$$
I\left(q_{x}, q_{y}\right)=\left|\sum_{n=-N / 2}^{N / 2} F\left(q_{x}, q_{y}\right)_{n} \exp \left(i q_{y} d_{y} n\right)\right|,
$$

with $N$ the number of layers and $d_{y}$ the distance between two close-packed layers. The expression can be expanded to obtain (omitting the $q_{x}$ and $q_{y}$ )

$$
I\left(q_{x}, q_{y}\right)=\sum_{n=-N / 2}^{N / 2} \sum_{m=-N / 2}^{N / 2} F_{n} F_{m}^{*} \exp \left[i q_{y} d_{y}(n-m)\right],
$$

which in effect sums all interactions between layers. We can apply a change of summation order by creating a summation parameter that sums over the difference $n^{\prime}=n-m$, which will replace one of the summation parameters. We can eliminate the other summation parameter by stating that all $N$ layers are equal, so that only the number of realizations of a certain difference between layers count. This leads to a factor $N-n^{\prime}$ that replaces the second summation. The next step is to replace the term $F_{n} F_{m}^{*}$ by an expression that represent the change of a certain half-layer pair combination occurring a distance $n^{\prime}$ from a given half-layer pair. Given a vector $\mathbf{v}$ holding the $F$ values for all half-layer pairs, and a matrix $P$ that gives the chance for a certain half-layer pair being followed by a certain second half-layer pair, the expression for the diffraction becomes

$$
\begin{aligned}
I\left(q_{x}, q_{y}\right)= & \sum_{n=1}^{N} \frac{1}{6} \mathbf{v} P^{n} \mathbf{v}^{*}(N-n) \exp \left(i q_{y} d_{y} n\right)+\frac{N}{6}|v|^{2} \\
& +\sum_{n=1}^{N} \frac{1}{6} \mathbf{v}\left(P^{T}\right)^{n} \mathbf{v}^{*}(N-n) \exp \left(-i q_{y} d_{y} n\right) .
\end{aligned}
$$

The matrix $P$ is rather sparse, as for every half-layer pair only two possibilities exist: either the stacking is f.c.c. (with chance $\alpha$ ) or it is hexagonally close packed (h.c.p., with chance $1-\alpha$ ). For $\alpha=0.5$, the value of $\mathbf{v} P^{n} \mathbf{v}^{*}$ is such that the equation above splits into two parts: one for orders $n_{x}$ of $q_{x}$ divisible by three, and one not divisible by three. For orders divisible by three,

$$
\begin{aligned}
I\left(q_{x}, q_{y}\right)= & \frac{N}{2}\left(\left|F_{A B}\right|^{2}+\left|F_{A C}\right|^{2}\right) \\
& +\frac{1}{2} \operatorname{Re}\left\{\sum_{n=1}^{N}\left|F_{A B}+F_{A C}\right|^{2} \Lambda_{N}\left[\exp \left(i q_{y} d_{y}\right)\right]\right\} \\
= & \left|F_{A B}+F_{A C}\right|^{2} \frac{\sin ^{2}\left(N q_{y} d_{y} / 2\right)}{4 \sin ^{2}\left(q_{y} d_{y} / 2\right)} \\
& +\frac{N}{4}\left|F_{A B}-F_{A C}\right|^{2}
\end{aligned}
$$

with $\Lambda_{N}$ the arithmetic geometric progression

$$
\begin{aligned}
\Lambda_{N}(x) & =\sum_{n=1}^{N}(N-n) x^{n} \\
& =\frac{x^{N+1}-N x^{2}+N x-x}{(x-1)^{2}} .
\end{aligned}
$$

Even for a low number of layers, the expression shows quite sharp peaks with periodicity $2 \pi q_{y} d_{y}$.

For the zeroth order in the $x$ direction, $F_{A B}=F_{A C}$, so it can be easily seen that irrespective of the value of the contrast $\psi$ (which is incorporated into $F_{A B}$ ), a secondary Bragg rod [i.e. a continuously distributed intensity between the sharp Bragg peaks (Petukhov et al., 2003)] will not appear.

For diffraction orders that are not divisible by three, the solution looks less elegant:

$$
\begin{aligned}
I\left(q_{x}, q_{y}\right)= & \frac{N}{2}\left(\left|F_{A B}\right|^{2}+\left|F_{A C}\right|^{2}\right) \\
& -\operatorname{Re}\left\{\left\{\left|F_{A B}\right|^{2} \exp \left(-i n_{x} 2 \pi / 3\right)+\left|F_{A C}\right|^{2} \exp \left(i n_{x} 2 \pi / 3\right)\right.\right. \\
& \left.\left.+2 \operatorname{Re}\left[F_{A B} F_{A C}^{*} \exp (-i n 2 \pi / 3)\right]\right\} \Lambda_{N}\left[-\exp \left(i q_{y} d_{y}\right) / 2\right]\right\},
\end{aligned}
$$

but when plotted, clearly shows a Bragg rod structure.

Instead of considering the diffraction pattern of stacking parameter $\alpha=0.5$, we can also look at the diffraction pattern for arbitrary $\alpha$ in the interval $[0,1]$. The values in the matrix $P$ take on rather complicated forms, but for orders $n_{x}$ divisible by three, the expression for $v P^{n} v^{*}$ takes on the relatively simple form of

$$
v P^{n} v^{*}=\frac{3}{2}\left[\left|F_{A B}+F_{A C}\right|^{2}+(2 \alpha-1)^{n}\left|F_{A B}-F_{A C}\right|^{2}\right],
$$




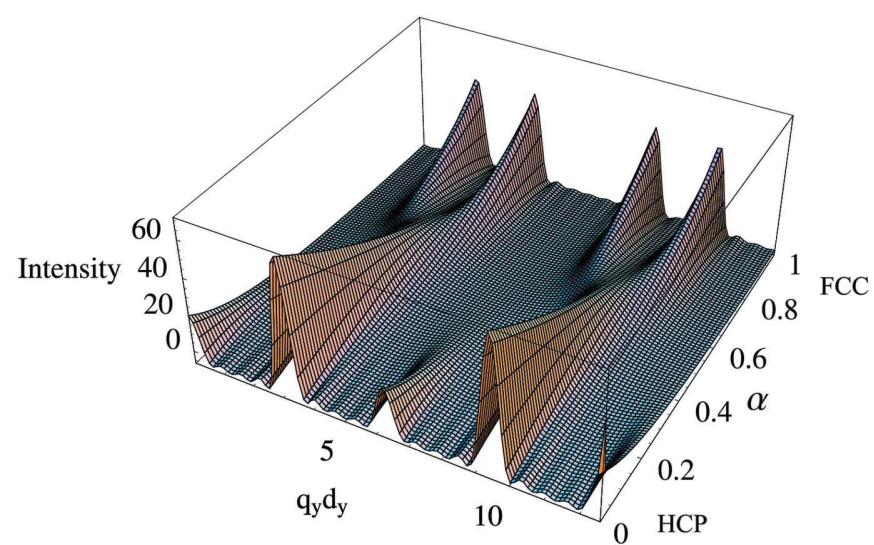

Figure 6

The structure of the Bragg rods for $F_{A B}=F_{A C}=1$, showing the transition between a pure f.c.c. and a pure h.c.p. stacking.

which changes equation (10) by adding a factor $\left|F_{A B}-F_{A C}\right|^{2} \operatorname{Re}\left\{\Lambda_{N}\left[(1-2 \alpha) \exp \left(i q_{y} d_{y}\right)\right]\right\} / 2$, which is also zero for $n_{x}=0$. For this order, no matter what the exact stacking sequence is, the intensity profile will always be identical.

Finally, for orders $n_{x}$ not divisible by three and arbitrary $\alpha$, we can write the expression for $v P^{n} v^{*}$

$$
\begin{aligned}
v P^{n} v^{*}= & \frac{3}{2} \frac{\left(\zeta_{+}^{n}+\zeta_{-}^{n}\right)}{2^{n}}\left(\left|F_{A B}\right|^{2}+\left|F_{A C}\right|^{2}\right) \\
& +\frac{3 \alpha}{2\left(\xi^{1 / 2}\right)} \frac{\left(\zeta_{-}^{n}-\zeta_{+}^{n}\right)}{2^{n}}\left[\exp \left(i n_{x} 2 \pi / 3\right)\right. \\
& \left.-\exp \left(-i n_{x} 2 \pi / 3\right)\right]\left(\left|F_{A B}\right|^{2}-\left|F_{A C}\right|^{2}\right) \\
& -6 \operatorname{Re}\left[\frac{1-\alpha}{\xi} \frac{\left(\zeta_{-}^{n}-\zeta_{+}^{n}\right)}{2^{n}} F_{A B} F_{A C}^{*} \exp \left(-i n_{x} 2 \pi / 3\right)\right]
\end{aligned}
$$

$$
\begin{gathered}
\xi=\left(4-8 \alpha+\alpha^{2}\right)^{1 / 2}, \\
\zeta_{+}=-\alpha+\xi \\
\zeta_{-}=-\alpha-\xi
\end{gathered}
$$

Because of the complexity of the final expression we will not give it here. We are able, however, to show the development of the Bragg rods by examining the influence of the terms $\left|F_{A B}\right|^{2}+\left|F_{A C}\right|^{2},\left|F_{A B}\right|^{2}-\left|F_{A C}\right|^{2}$ and $\operatorname{Re}\left[F_{A B} F_{A C}^{*} \exp \left(-i n_{x} 2 \pi / 3\right)\right]$. If we evaluate the above expression for $F_{A B}=F_{A C}=1$, we obtain a result (as can be seen in Fig. 6) that is identical to the results found in the treatment of Wilson (1949). The minus and real-part terms adjust the structure of the peaks, where the minus term has the most influence on the structure of the f.c.c. side $(\alpha>1 / 2)$ and the real-part term mostly influences the h.c.p. side $(\alpha<1 / 2)$.

\section{Conclusion}

We have shown that the phase-only WKB approximation is a suitable tool to model dynamic diffraction in colloidal crystals. The model is consistent with diffraction theory for low contrasts and offers a satisfying explanation for the occurrence of high-magnitude diffraction peaks found earlier (Petukhov et al., 2004).

However, not all effects can be explained by the theory. It is lacking in the explanation of the occurrence of secondary Bragg rods (Petukhov et al., 2003), which presumably originate from dynamic effects for which the model is not suited. First of all, the present model does not take the curvature of the Ewald sphere into account, which may play a role. Furthermore, the present model deals only with ideal colloidal crystals and neglects possible lattice disorder. For example, the effect of the particle fluctuations around the ideal lattice positions on the Bragg peak intensity and the diffuse scattering (Slama et al., 2006) in the strongly dynamic diffraction can significantly deviate from the result of the Debye-Waller theory.

The authors thank Sylvie Roke, Gert Jan Vroege and Henk Lekkerkerker for fruitful discussion. The present work was encouraged by results collected in several experiments at the DUBBLE beamline BM-26 at the European synchrotron radiation facility (ESRF) in Grenoble. The Netherlands Organization for the Advancement of Research (NWO) is acknowledged for providing us with the possibility of performing these measurements. The staff of the beamline and the members of the experimental teams are gratefully acknowledged. In particular, we would to thank Igor Dolbnya, Xinyi Xian and Dirk Aarts for their important contributions.

\section{References}

Asher, S. A., Holtz, J., Weissman, J. \& Pan, G. S. (1998). MRS Bull. 23(10), 44-50.

Auer, S. \& Frenkel, D. (2003). Phys. Rev. Lett. 91, 015703(1-4).

Blaaderen, A. van, Ruel, R. \& Wiltzius, P. (1997). Nature (London), 385, 321-324.

Blanco, A., Chomski, E. \& Grabtchak, S. (2000). Nature (London), 405, 437-440.

Bolhuis, P. G., Frenkel, D., Mau, S. C. \& Huse, D. A. (1997). Nature (London), 388, 235-236.

Cheng, Z., Chaikin, P. M., Zhu, J., Russel, W. B. \& Meyer, W. V. (2002). Phys. Rev. Lett. 88, 015501(1-4).

Davidchack, R. L. \& Laird, B. B. (2000). Phys. Rev. Lett. 85, 45714574.

Deirmendjian, D. (1957). Ann. Geo. 13, 286-306.

Drakopoullos, M., Snigirev, A., Snigireva, I. \& Schilling, J. (2005). Appl. Phys. Lett. 86, 014102(1-3).

Dux, C. \& Versmold, H. (1997). Phys. Rev. Lett. 78, 1811-1814.

Elliot, M. S., Haddon, S. B. \& Poon, W. C. K. (2001). J. Phys. Condens. Matt. 13, L553-L558.

Feigin, L. A. \& Svergun, D. I. (1987). Structure Analysis by SmallAngle $X$-ray and Neutron Scattering. New York: Plenum Press.

Gasser, U., Weeks, E. R., Schofield, A., Pusey, P. N. \& Weitz, D. A. (2001). Science, 292, 258-262.

Harland, J. L. \& van Megen, W. (1997). Phys. Rev. E, 55, 3054-3067. Henderson, S. I. \& van Megen, W. (1998). Phys. Rev. Lett. 80, 877880.

Hoogenboom, J. P., van Landen-Suurling, A. K., Romijn, J. \& van Blaaderen, A. (2003). Phys. Rev. Lett. 90, 138301(1-4).

Hoover, W. G. \& Ree, F. H. (1968). J. Chem. Phys. 49, 3609-3617.

Hulst, H. C. van de (1981). Light Scattering by Small Particles. New York: Dover.

Imhof, A. \& Pine, D. J. (1997). Nature (London), 389, 948-951.

James, R. W. (1965). The Optical Principles of the Diffraction of $X$-rays. Ithaca, NY: Cornell University Press. 
Kegel, W. K. \& Dhont, J. K. G. (2000). J. Chem. Phys. 112, 3431-3436. Liu, J., Weitz, D. A. \& Ackerson, B. J. (1993). Phys. Rev. E, 48, 1106. Martelozzo, V. C., Schofield, A. B., Poon, W. C. K. \& Pusey, P. N. (2002). Phys. Rev. E, 66, 021408(1-14).

Megens, M. \& Vos, W. L. (2001). Phys. Rev. Lett. 86, 4855-4858.

Petukhov, A. V., Aarts, D. G. A. L., Dolbnya, I. P., de Hoog, E. H. A., Kassapidou, K., Vroege, G. J., Bras, W. \& Lekkerkerker, H. N. W. (2002). Phys. Rev. Lett. 88, 208301(1-4).

Petukhov, A. V., Dolbnya, I. P., Aarts, D. G. A. L. \& Vroege, G. J. (2004). Phys. Rev. E, 69, 031405(1-10).

Petukhov, A. V., Dolbnya, I. P., Aarts, D. G. A. L., Vroege, G. J. \& Lekkerkerker, H. N. W. (2003). Phys. Rev. Lett. 90, 028304(1-4).

Petukhov, A. V., Thijssen, J. H. J., 't Hart, D. C., Imhof, A., van Blaaderen, A., Dolbnya, I. P., Snigirev, A., Mossaïd, A. \& Snigireva, I. (2006). J. Appl. Cryst. 39, 137-144.

Polson, J. M., Trizac, E., Pronk, S. \& Frenkel, D. (2000). J. Chem. Phys. 112, 5339-5342.

Pronk, S. \& Frenkel, D. (1999). J. Chem. Phys. 110, 4589-4592.

Pronk, S. \& Frenkel, D. (2003). Phys. Rev. Lett. 90, 255501(1-4).

Pusey, P. N. \& van Megen, W. (1986). Nature (London), 320, 340-342.

Pusey, P. N., van Megen, W., Bartlett, P., Ackerson, B. J., Rarity, J. G. \& Underwood, S. M. (1989). Phys. Rev. Lett. 63, 2753-2756.

Rengarajan, R., Jiang, P., Larrabee, D. C., Colvin, V. L. \& Mittleman, D. M. (2001). Phys. Rev. B, 64, 205103(1-4).

Roke, S., Bonn, M. \& Petukhov, A. V. (2004). Phys. Rev. B, 70, 115106(1-10).
Sakurai, J. J. (1994). Modern Quantum Mechanics, 2nd ed. Reading, Mass.: Addison-Wesley

Sanders, J. V. (1968). Acta Cryst. A24, 427.

Schaetzel, K. \& Ackerson, B. J. (1993). Phys. Rev. E, 48, 3766-3777.

Slama, S., von Cube, C., Kohler, M., Zimmermann, C. \& Courteille, P. W. (2006). Phys. Rev. A, 73, 023424(1-9).

Velikov, K. P. (2002). Science, 296, 106-109.

Verhaegh, N. A. M., van Duijneveldt, J. S., van Blaaderen, A. \& Lekkerkerker, H. N. W. (1995). J. Chem. Phys. 102, 1416.

Versmold, H., Musa, S. \& Bierbaum, A. (2002). J. Chem. Phys. 116, 2658-2662.

Vlasov, Y. A., Bo, X. Z., Sturm, J. C. \& Norris, D. J. (2001). Nature (London), 414, 289-293.

Vos, W. L., Megens, M., van Kats, C. M. \& Bosecke, P. (1997). Langmuir, 13, 6004-6008.

Wijnhoven, J. E. G. J., Bechger, L. \& Vos, W. L. (2001). Chem. Mater. 13, 4486-4499.

Wijnhoven, J. E. G. J. \& Vos, W. L. (1998). Science, 281, 802-804.

Wilson, A. J. C. (1949). X-ray Optics. London: Methuen.

Yethiraj, A. \& van Blaaderen, A. (2003). Nature (London), 421, 513517.

Zachariasen, W. H. (1946). Theory of X-ray Diffraction in Crystals. New York: Wiley.

Zhu, J., Li, M., Rogers, R., Meyer, W., Ottewill, R. H., Crew, S.-S. S., Russel, W. B. \& Chaikin, P. M. (1997). Nature (London), 387, 883885 . 